\title{
Utility of Short Message Service (SMS) for Remote Data Collection for HIV in Low- and Middle-Income Countries
}

\author{
Alison L. Drake ${ }^{1} \cdot$ Claire Rothschild $^{2} \cdot$ Wenwen Jiang $^{2} \cdot$ Keshet Ronen $^{1} \cdot$ Jennifer A. Unger ${ }^{1,3}$ \\ Accepted: 15 September 2020 / Published online: 3 October 2020 \\ (C) Springer Science+Business Media, LLC, part of Springer Nature 2020
}

\begin{abstract}
Purpose of Review Short message system (SMS) communication is widespread in low- and middle-income countries (LMICs), and may be a viable approach to address challenges with in-person data collection for HIV-related research and monitoring and evaluation. We reviewed the literature to characterize potential benefits and challenges with using SMS for remote data capture, including examples from HIV and sexual and reproductive health.

Recent Findings In our review, we found that studies that have used SMS to capture sensitive, self-reported data found this was an acceptable and feasible strategy, and may reduce social desirability bias of self-reported data; but studies are limited. Shared phones and privacy concerns have been described as challenges, but can be addressed with enhanced security features. Response rates to SMS surveys varied significantly by topic, population, and setting.

Summary SMS may improve generalizability and precision of health and behavior data for HIV in research and programs, but use in LMICs is limited. SMS systems should be carefully designed to overcome potential implementation hurdles.
\end{abstract}

Keywords SMS $\cdot$ Text messages $\cdot$ Mobile phone $\cdot$ Remote data collection $\cdot$ HIV $\cdot$ Sexual and reproductive health

\section{Introduction}

Digital approaches to public health problems, including mobile and web applications, short message system (SMS), and tablet and other computer-assisted devices, are becoming commonplace. In low- and middle-income countries (LMICs), mobile health (mHealth) technology has been recognized as a powerful tool to improve HIV outcomes and care delivery. Globally, over 5 billion people have access to mobile phones; and coverage, acceptability of technology, and familiarity with use are anticipated to only increase over time [1]. Global funding agencies are making strategic investments in

This article is part of the Topical Collection on eHealth and HIV

Alison L. Drake

adrake2@uw.edu

1 Department of Global Health, University of Washington, 3980 14th Ave NE, WU Box 351620, Seattle, WA 98195, USA

2 Department of Epidemiology, University of Washington, 3980 14th Ave NE, UW Box 351619, Seattle, WA, USA

3 Department of Obstetrics and Gynecology, University of Washington, Seattle, WA, USA mobile technology approaches that provide innovative solutions to these problems, including improving HIV prevention, care, and treatment [2-5]. Despite the potential for technology to propel global HIV initiatives, there is substantial variability in the quality, fidelity, and implementation of strategies using mobile technology. The World Health Organization (WHO) currently recommends use of mHealth technologies to strengthen health systems yet also recognizes the need for guidance on optimizing use of technology and has developed policy documentation on best practices [6].

High-quality data capture is essential to advance novel HIV research, as well as monitor progress of programs designed to prevent and treat HIV. Yet, there are many obstacles to in-person data collection, including logistical constraints that exclude individuals who face challenges with accessing in-person visits, as well as social desirability bias for sensitive questions. As a result, in-person data collection may lead to less precise or incomplete ascertainment of exposures and outcomes. Simple and widespread technology, such as SMS, could be a viable option to improve collection of a variety of health and behavior data for HIV research and programs. This approach is particularly appealing when in-person data collection is difficult, including during the COVID-19 pandemic. 
With the expansion of mobile phone coverage in LMICs, SMS was one of the first mHealth technologies used to support health and delivery of health care services. SMS were particularly attractive in LMICs and have been used to complement clinical HIV prevention, care, and treatment programs. SMS use remains popular in settings where penetration of smartphones and internet access is low, and more sophisticated digital tools, such as mobile applications ("apps"), have limited reach. They have been used to test approaches for disseminating HIV education messages and encouraging HIV testing [7-13], providing counseling support to patients and triaging their care-seeking decisions [13-20], providing reminders for clinic visits and medication [11, 13, 17-22], and offering an alternative strategy for laboratories to share test results with providers [23]. Several trials and evaluations have found SMS sent to patients improve antiretroviral therapy (ART) adherence and HIV virologic suppression [11, 13, 21, 22, 24, 25], rates of early infant testing for HIV and postpartum prevention of mother-to-child HIV transmission (PMTCT) retention $[8,26]$, and retention in HIV care $[11,21,26-28]$. However, efforts to use SMS to remotely capture data from individuals to measure HIVrelated risks, behaviors, and clinical outcomes in the context of research or programmatic monitoring and evaluation are limited.

In this review, we examine potential benefits of, and challenges with, using SMS for remote HIV-related data capture. We consider SMS data capture in the context of research and health programs in LMICs, and present examples where SMS have been used for remote data capture in the fields of HIV and sexual and reproductive health. We collate lessons learned from these studies, as well as from fields outside of HIV and sexual and reproductive health, and identify areas where SMS has enormous potential to improve data collection in the context of research and program delivery, and propose guidelines for best practices.

\section{Methods}

We searched titles and abstracts published on PubMed through December 2019 using terms to identify mobile health, SMS, and text messaging; HIV or sexual and reproductive health; and in combination with terms for data collection and/or surveys. We also conducted a similar search using the Google search engine. We reviewed citations with relevant topics in articles identified from the PubMed and Google search. Articles were reviewed if they were primary research articles that included mHealth technology for data collection; while we prioritized articles from LMIC on HIV and sexual and reproductive health, we also included articles outside of this setting if they contributed information about barriers or challenges to implementing remote data collection using mHealth technology, prioritizing data from LMIC whenever possible. Review articles of mHealth or SMS, and HIV, that included studies from LMICs were also included. As thematic areas to include in the review were identified, PubMed and Google searches were conducted again to identify additional articles with terms for these themes. Finally, we also reviewed relevant articles and guidance documents the research team was previously aware of.

\section{Results}

Overall, 54 references were included in our review: 33 primary research articles, 18 review articles, and 3 guidance documents. The majority of references $(n=47,87 \%)$ included HIV and sexual and reproductive health results or themes; 32 were identified through searches and 22 the research team was aware of. Major thematic areas included phone access and privacy, timing of SMS delivery and response $(n=5)$, behavior change or behavior change theory $(n=4)$, SMS use for routine activities $(n=1)$, data collection on sensitive topics $(n=10)$, response rates $(n=7)$, phone access and privacy $(n=23)$, data quality $(n=8)$, generalizability $(n=2)$, and ecological momentary assessment (EMA, $n=1$ ).

\section{Overcoming Logistical Constraints to In-person Data Collection}

Remote data capture using SMS technology has the potential to overcome many limitations of in-person data collection efforts for research or program monitoring. This approach offers the opportunity to provide real-time monitoring of behaviors and health outcomes, to shorten time intervals between measurements and prevent missing data at scheduled visits, and more accurately capture dynamic behavioral constructs. One example of real-time monitoring is EMA, which permits repeated reporting of behaviors in the moment and environment in which they occur, and has been used for a variety of HIV-related research questions in the USA [29]. SMS could be used to support EMA, as well as a wider range of experiences that change over longer intervals of time. Twoway SMS can capture these changes both by SMS system programming to tailor the time interval between subsequent SMS soliciting responses and permitting individuals to initiate a report of a change through SMS without being prompted. Thus, the capacity for real-time monitoring reduces recall bias associated with longer reporting intervals common in prospective research studies and between visits for clinical care, and may improve measurement precision of health behaviors and outcomes in research and programs. 
Many SMS studies have customized the timing of SMS delivery based on individual preferences [20,30-32], in an effort to increase the likelihood SMS are received at a day and time that is convenient. While most SMS that request a response are replied to immediately after receipt [33, 34], SMS are easier, and require less time to respond to, than a phone call or voicemail. Unlike phone calls, recipients can screen SMS for the effort required to respond. Fluidity in the timing of interaction with SMS facilitates engagement by recipients at times that are convenient, increasing opportunities for participation. Responding to SMS inquiries also provides a privacy advantage over phone calls, as SMS responses can be replied to discreetly while verbal responses to phone inquiries can be overheard and present a different level of vulnerability [35]. SMS are readily accessible for those with mobile phone access, as phones are integrated into daily life for regular communication, including banking and engaging in social media [36].

SMS systems can be low cost [37] and less expensive to collect self-reported survey data than in-person strategies due to reduced personnel. They save both patient and provider time required for visits to the facility that do not require clinical care, evaluation, or sample collection. In times when services are disrupted and travel to facilities is unsafe or not possible due to political unrest, natural disasters, health care worker strikes, or most recently a global COVID-19 pandemic, SMS can fill a critical void in monitoring and evaluation efforts, as well as service provision.

\section{Acceptability and Response Rates}

SMS can improve generalizability of study findings, and reduce selection bias, by including individuals who are often left out of research because they are unable to access care, live in remote settings, are too sick to visit health care facilities, or lack resources to pay for transportation-related expenses and health care services required to participate in facility-based research or receive follow-up care [38]. Near-universal access to SMS technology may also improve generalizability, with higher external validity compared with data collection using smartphones in populations with low to moderate smartphone penetration $[38,39]$. Ascertainment of data that can be selfreported through SMS has potential to increase retention rates if individuals are unable to navigate logistical barriers required for facility-based research, including employment, family duties, or relocation, but are still interested in and able to respond to SMS. For example, reaching marginalized populations at high risk of HIV infection is challenging in HIV prevention research, and maximizing enrollment and high retention is critical to accurately measure success and failures for those involved in prospective studies or programs. In a preexposure prophylaxis (PrEP) demonstration project in Kenya and Uganda conducted in 2014 that asked participants to report sexual behavior and PrEP adherence, $95 \%$ of participants said SMS were easy to use, $74 \%$ had no challenges with SMS, and $72 \%$ preferred SMS over in-person study visits [40•0]. Growing familiarity and comfort with using this technology make SMS increasing accessible.

While SMS have been described as generally simple and easy to use $[38,40 \cdot \bullet$, prior studies that have used remote SMS messages and surveys have found variable response rates. In a large study of SMS for ART adherence in Kenya, response rates to two-way message inquiries were as high as 69\% [41]. Another Kenyan study that captured fertility data among HIV-serodiscordant couples through a daily, short SMS survey found $78 \%$ of women completed all SMS, $7 \%$ partially completed, and $16 \%$ did not complete any surveys [42•]. They also noted seasonal variation in responses, with lower response rates over the end-of-year holidays [42•]. In Uganda, a communitybased survey with multiple topics found an overall response rate of $70 \%$, with the highest response rate for surveys on $\mathrm{HIV} / \mathrm{sexually}$ transmitted diseases (STDs) (79\%), followed by sexual behavior (64\%), male medical circumcision (56\%), and family planning (53\%); notably only $37 \%$ completed demographic survey questions [43]. Median response time was $50 \mathrm{~min}$ and declined over time, even with SMS reminders with potential for incentives (prizes, phone credit, or free HIV testing) [43]. In contrast, a 2010 study on ART adherence among caregivers of HIV-infected children that used SMS for reporting found that despite high initial interest and participation, weekly completion of SMS surveys was low (range 17-33\%); however, confusion around use of a personal identification number (PIN) for security issues was cited as a potential explanation for low completion rates [44]. Similarly, in a large, free, opt-in platform that provides information on family planning by SMS (Mobiles for Reproductive Health, m4RH), only $39.5 \%$ of registered users responded to a 5-6-item survey about their knowledge and use of contraception, and only $20.9 \%$ completed all survey questions [34]. Approaches to mitigate respondent fatigue may be warranted for studies planning on using SMS data collection, as attrition is likely to occur over time [45].

Few studies have directly compared performance of different telecommunication platforms for remote data capture in research studies and clinical care. In Peru and Honduras, response rates were compared using interactive voice response (IVR), computer-assisted telephone interview (CATI), and SMS in a population-based representative sample of households in a Gallup Poll commissioned by the World Bank, measuring household characteristics. SMS and IVR had similar response and retention rates (21\% vs. $19 \%$ in Peru, $40 \%$ vs. $38 \%$ in Honduras; respectively) but lower rates than CATI 
(39\% Peru, 72\% Honduras) [46]. These results concur with generally low initial response rates for population-based sampling approaches (as opposed to generally higher nonpopulation-based approaches) and suggest there was some differential attrition by data collection method. However, assignment to data collection method also varied by country (randomized approach in Peru vs. multiple strategies used among households who initially participated in-person in Honduras) [46].

In some SMS studies, participation rates were high when economic incentives were offered. In the Gallup Poll survey in Peru and Honduras that directly compared attrition by mode of data collection, economic incentives led to moderate reductions in attrition; attrition was reduced by $5-10 \%$, but costs were threefold higher for IVR and fivefold higher for CATI compared with SMS in Peru [46]. While many studies use incentives to encourage participation in SMS surveys, few studies have determined the impact in randomized studies. One study in Kenya on family planning found incentives had no effect on response rates [34].

\section{Challenges with Implementation}

\section{Consistency in Phone Access and Privacy}

While SMS have several features that make them appealing to incorporate into HIV research and programs for data collection, there are limitations to using this technology which require special considerations in LMICs to optimize utility. Despite high and expanding coverage of mobile phone access, shared phones and having multiple phones and/or SIM cards are common in some settings, creating barriers to using SMS for remote data capture. Phones may be shared within a household among couples or with other family members, and access to phones may be variable throughout the day or week [47]. Sharing phones has implications for privacy, as individuals who share phones may need to take extra precautions to delete incoming and outgoing SMS that contain sensitive information [48]. This includes data related to their HIV status, use of medication, and participation in research. Women are more likely to share phones with their partner and have more inconsistent access to phones than men. In addition, use of shared phones by health care workers has also led to problems. For example, in Zimbabwe, HIV viral load results were shared with facilities by SMS, but instructions were not provided to all health care workers who shared the phone creating confusion about what to do with information in the SMS $[15,49]$. Other studies have reported that health care workers in LMICs use their mobile devices for informal activities both related, and unrelated, to their jobs [50]. This additional use may increase risk of breaches in confidentiality, as well as opportunities for devices to be lost, broken, or stolen.
Several strategies have been implemented to minimize potential breaches in confidentiality and maintain privacy. SMS participants can "opt-in" to receive more detailed messages, such as overt messaging about HIV and use of medication [51]. They can also be advised to delete any messages received, or sent, that contain sensitive information. PINs or passwords to unlock SMS or screens have also been used to maintain security of sensitive information [52], but may create a barrier to participation [44].

Access to participating in data collection efforts using SMS can also be hampered by electric power disruptions and damaged or broken phones [53, 54]. In Burkina Faso, 65\% of pregnant women living with HIV in a study that used SMS for appointment reminders had phones that were damaged and needed to be replaced, some due to extreme weather conditions [49]. While phones were provided to participants in many early SMS studies, it is increasingly less common to do so as frequency of phone ownership has increased [35] and due to concerns that phone provision may not increase participation if phones may be sold or used for other purposes [55]. As a result, phone provision was not determined to be a scalable or sustainable approach to digital health programs $[39,56]$. However, in areas where mobile phones and SMS literacy are not ubiquitous, SMS engagement may be suboptimal [57] and training can result in substantial start-up costs and delays [49]. In some areas, phone credit is required and creates a barrier to sending and responding to SMS [48, 58]. Reverse-billed short codes are a common approach to mitigate requirements for phone credit [14, 59-62], as is a "flashback" where the SMS participant calls the sender to trigger a call back to avoid costs incurred by participants [63]; both alleviate participant concerns about cost of responding to SMS. Inconsistent power supplies, limited network range and strength, and network outages have also been previously reported $[49,54,64,65]$. SMS systems operated through a web-interface may also experience outages when internet service is disrupted $[48,54,58]$. The type, frequency, and duration of these issues in individual communities or countries should be considered during planning stages.

\section{Errors Due to Phone Quality and Language}

The quality of data collected through SMS is related to the type of data collected. Remote data capture using SMS can capture free-form, open response text or coded alphanumeric responses. Similar to paper or other electronic surveys, freeform text offers the most flexibility, including descriptive narrative responses within the SMS character limit, but is the most difficult data to aggregate and analyze. In contrast, coded responses can be used to select discrete answers, including dichotomous yes/no responses, but require additional questions to capture multiple answers. Discrete data capture is 
significantly easier to clean and analyze, and may also incur lower labor costs for data cleaning and analysis activities compared with free-form text responses. It also provides an opportunity to develop skip patterns based on prior responses. This smart logic response approach, with limited, coded numerical responses for SMS inquiries, has been tested in several studies [53, 66-68]. Deploying SMS on a platform with "longitudinal memory," where follow-up SMS inquiries sent minutes, days, or weeks after a response can resume a skip pattern from a prior SMS survey provides even more flexibility. While fewer keystrokes with coded responses provide fewer opportunities for data entry error, they are less forgiving and errors are more difficult to find and detect than free-form text.

As a result, the mobile phone interface, keyboard type, screen size, and ability for respondents to accurately enter responses may also reduce quality and accuracy of data collection. If coded responses are erroneously entered and submitted by SMS, it is not possible to recall the SMS and resubmit, or otherwise "undo" an entry. Use of a second, confirmatory SMS inquiry for coded, one-character responses (rather than free-form text) is one strategy that has been used to reduce data entry errors and confirm critical information captured through SMS. However, this approach should be balanced with attention to respondent fatigue during the survey, to maintain engagement by the end user. Two-way systems have also been set up to accept multiple potential responses [69].

In addition, in countries with multiple languages, literacy may vary by language, and considerations for handling multiple language responses are necessary [54]. Unique considerations and accommodations may be necessary if nonLatin alphabets on phones are used. If multiple languages are common, SMS can be offered in multiple language tracks if investigators or programs are prepared to interpret responses that may be in multiple languages or a hybrid of languages [54], such as "Sheng"-a combination of Swahili and English; this may include interpretation manually or by computer software. It is worth noting that while relatively advanced natural language processing software tools exist to interpret languages such as English, far fewer tools have been developed for the indigenous languages spoken in subSaharan Africa where the HIV epidemic is most concentrated [70]. Alternatively, SMS can be limited to only support specific languages. This approach may be warranted if the additional languages contribute relatively little data, generalizability is similar with and without the additional language, or due to logistical constraints to support multiple languages. These decisions can have implications for the 160-character count limit for SMS [37]. SMS length will vary by language, and while an initial SMS may be within the character limit, translated versions into other languages may exceed the limit, necessitating two SMS to accommodate the longer translated version.

\section{SMS for Data Collection on Sensitive Topics}

The validity and reliability of self-reported responses on sensitive topics, including sexual behavior, is the subject of much debate, with many hypothesizing that social desirability bias leads to underreporting of behaviors in face-to-face interviews [71-73]. To overcome underreporting and discrepancies between behavioral risk factors and HIV/sexually transmitted infection (STI) acquisition in LMICs, a variety of remote, self-administered, or computer-assisted surveying modes (IVR, CATI, self-administered surveys, and web surveys) [74] have been used, yet SMS surveys as a specific modality have rarely been used to collect this type of data. A 2010 systematic review of alternative interview modes for capturing information on sensitive HIV risk behaviors failed to identify any published studies comparing SMS data collection to inperson surveys [75]. It is plausible that remote SMS surveys may be more acceptable and result in higher reporting of a variety of sensitive behaviors, such as sexual activity and adherence to novel biomedical HIV prevention interventions, compared to in-person surveys, similar to findings from other computer-assisted surveys $[71,73,75,76]$. SMS technology may also be preferable over many other modalities that perpetuate the digital divide by preferentially sampling participants with internet access, while excluding those who do not.

Studies to date suggest SMS surveys are both feasible and acceptable as a means for collecting information on sensitive HIV-related exposures and outcomes remotely. In a study assessing a mobile intervention to support safe conception among Kenyan serodiscordant couples, nearly $80 \%$ of all daily SMS surveys on fertility indicators (such as menses) and sexual activity were completed [42•]. Participants found the SMS format easy to use, and few expressed concerns about confidentiality in qualitative interviews [42•]. Another study of adherence to HIV PrEP in Kenya found that participants were willing to report sensitive behaviors via an SMS survey, with high completion rates (median 2 of 60 unanswered daily surveys); however, unanswered surveys were more common at the end of the study [52]. Nearly 50\% reported unprotected sex and 70\% reported at least one missed dose of PrEP [52]. Similarly, in a PrEP implementation study of Kenyan women, use of SMS to improve ascertainment of male partner HIV self-testing outcomes led to substantial increases in completeness of information [77]. In addition, women were more likely to report harm or negative partner reactions as a result of the HIV self-test by SMS compared with in-person reports, which may indicate increased willingness to report sensitive information remotely. Together, these data suggest SMS are an acceptable format to obtain sensitive HIV-related health behaviors and outcomes, and may bolster participation in research; however, evaluations directly comparing data collection modalities in the same study, population, and setting in LMICs are still needed. 


\section{Incorporating Health Behavior Theory}

A prior review of health behavior theory and its application to eHealth HIV interventions and research advocated for theory to be incorporated in the design [78], and these findings are relevant for SMS approaches for data collection. While SMS may be used solely as an approach to capture data, it can simultaneously be used to serve as an intervention designed to elicit behavior change. Elements of SMS interventions that have previously been shown to be successful for behavioral change outcomes such as interactivity, optimized frequency, and personalization [32, 37, 79-81] will also be important for high engagement, low attrition, and successful data collection efforts.

\section{Recommendations}

Based on our review, we have summarized key considerations in selecting and designing an SMS system for data collection in LMICs (Table 1), and identified 2 broad areas that have high potential to benefit from SMS as an approach to collect HIV-related data:

1. Surveillance. SMS could be a powerful tool to monitor disease HIV burden, health behaviors, or health outcomes. Surveillance efforts to conduct in-person assessments and manage data are labor intensive, and likely more costly than SMS. In addition, the ability to tailor frequency of monitoring to suit surveillance needs, and avoid limitations of collecting data only among individuals who seek and remain in care, makes SMS a good candidate for surveillance.

2. Monitoring self-reported outcomes. SMS promote ascertainment of self-reported outcomes for research or monitoring and evaluation activities for those who are doing well, capturing data on individuals who less frequently access in-person care than patients who require medical evaluations. This strategy could be important in supporting differentiated care models with longer intervals between visits to the health care facility for redosing of ART or other medications, or to check on outcomes that can be self-reported (such as ART adherence or side effects). It can also be used to more accurately measure ART or PrEP adherence, retention in care, and mortality, potentially capturing individuals transferring care to new facilities who may otherwise be misclassified as lost to follow-up when they are not. Finally, it could be used to support tele-triage, guiding clinical decisionmaking about the necessity of seeking care.
Table 1 Considerations for SMS-based data collection in low- and middle-income countries

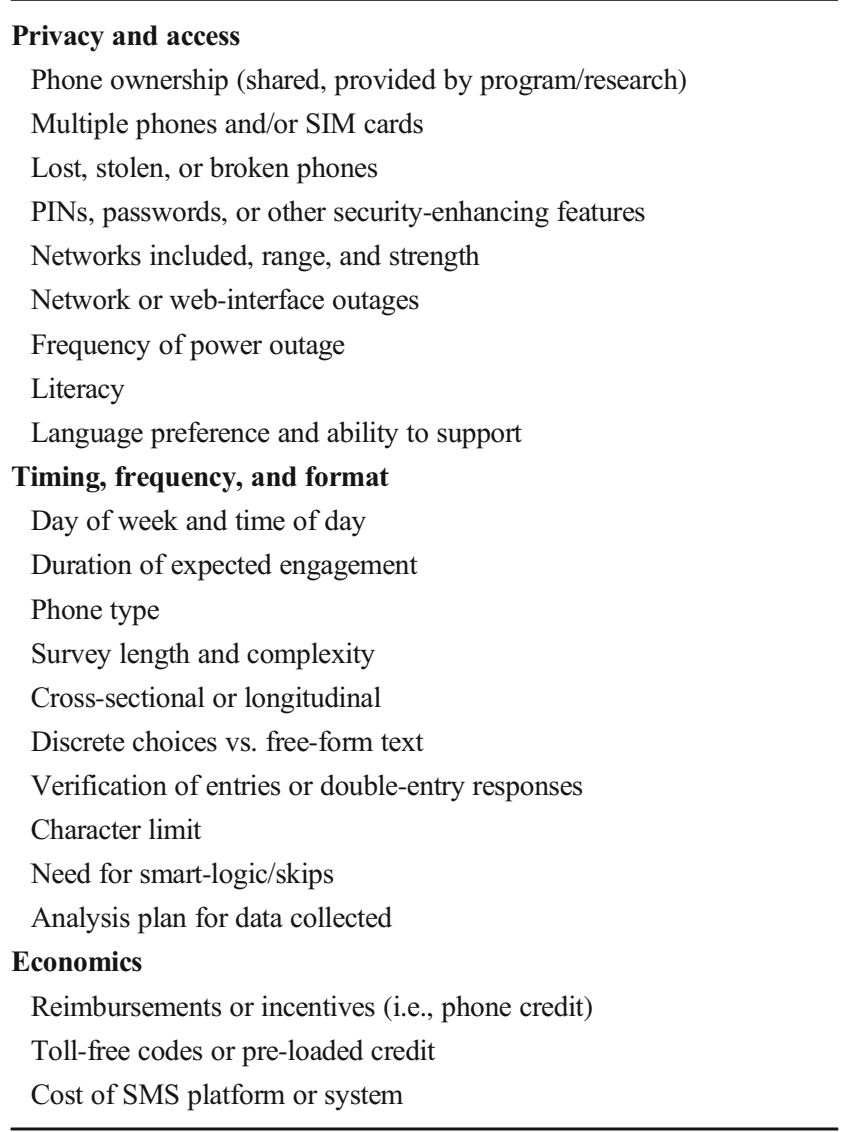

\section{Conclusions}

In conclusion, SMS have many appealing features that make them well suited to serve as a tool to collect data for HIV research and monitoring and evaluation activities, but are currently underutilized in LMICs. SMS can be successfully deployed, but logistical aspects of implementation should be carefully considered to avoid common pitfalls during the design stage, including assessments of who may be left out of an SMS-based approach. Researchers and programs should consider using SMS as strategy to improve quality of HIV-related data, including conducting robust evaluations of benefits and risks this approach.

Author Contributions ALD, CR, WJ, KR, and JU contributed to the literature search. All authors contributed to writing the manuscript and interpreting the results.

Funding This study was funded by NIH/NIAID P30-AI027757, NIH/ NIAID K01 AI1 16298 (ALD), and NIH/NICHD F31HD097841 (CR). 


\section{Compliance with Ethical Standards}

Conflict of Interest The authors declare that they have no competing interests.

\section{References}

Papers of particular interest, published recently, have been highlighted as:

- Of importance

•- Of major importance

1. Smartphone ownership is growing rapidly around the world, but not always equally: Pew Research Center; February 2019 [Available from: https://www.pewresearch.org/global/2019/02/05/ smartphone-ownership-is-growing-rapidly-around-the-world-butnot-always-equally/.

2. Catalani C, Philbrick W, Fraser H, Mechael P, Israelski DM. mHealth for HIV treatment \& prevention: a systematic review of the literature. Open AIDS J. 2013;7:17-41.

3. Horvath T, Azman H, Kennedy GE, Rutherford GW. Mobile phone text messaging for promoting adherence to antiretroviral therapy in patients with HIV infection. Cochrane Database Syst Rev. 2012;3: CD009756.

4. Gimbel S, Kawakyu N, Dau H, Unger JA. A missing link: HIV-/ AIDS-related mHealth interventions for health workers in low- and middle-income countries. Curr HIV/AIDS Rep. 2018;15(6):414 22.

5. Cooper V, Clatworthy J, Whetham J, Consortium E. mHealth interventions to support self-management in HIV: a systematic review. Open AIDS J. 2017;11:119-32.

6. WHO guideline: recommendations on digital interventions for health system strengthening. Geneva; 2019. Contract No.: Licence: CC BY-NC-SA 3.0 IGO.

7. United N. ECOSOC 'Texting 4 Health campaign'; Jinja, Uganda; June 2009.

8. Odeny TA, Bukusi EA, Cohen CR, Yuhas K, Camlin CS, McClelland RS. Texting improves testing: a randomized trial of two-way SMS to increase postpartum prevention of mother-tochild transmission retention and infant HIV testing. AIDS. 2014;28(15):2307-12.

9. Burton J, Brook G, McSorley J, Murphy S. The utility of short message service (SMS) texts to remind patients at higher risk of STIs and HIV to reattend for testing: a controlled before and after study. Sex Transm Infect. 2014;90(1):11-3.

10. Dryden-Peterson S, Bennett K, Hughes MD, Veres A, John O, Pradhananga R, et al. An augmented SMS intervention to improve access to antenatal CD4 testing and ART initiation in HIV-infected pregnant women: a cluster randomized trial. PLoS One. 2015;10(2):e0117181.

11. Taylor D, Lunny C, Lolic P, Warje O, Geldman J, Wong T, et al. Effectiveness of text messaging interventions on prevention, detection, treatment, and knowledge outcomes for sexually transmitted infections (STIs)/HIV: a systematic review and meta-analysis. Syst Rev. 2019;8(1):12.

12. Njuguna N, Ngure K, Mugo N, Sambu C, Sianyo C, Gakuo S, et al. The effect of human immunodeficiency virus prevention and reproductive health text messages on human immunodeficiency virus testing among young women in rural Kenya: a pilot study. Sex Transm Dis. 2016;43(6):353-9.

13. Demena BA, Artavia-Mora L, Ouedraogo D, Thiombiano BA, Wagner N. A systematic review of mobile phone interventions
(SMS/IVR/calls) to improve adherence and retention to antiretroviral treatment in low-and middle-income countries. AIDS Patient Care STDs. 2020;34(2):59-71.

14. Drake AL, Unger JA, Ronen K, Matemo D, Perrier T, DeRenzi B, et al. Evaluation of mHealth strategies to optimize adherence and efficacy of option B+ prevention of mother-to-child HIV transmission: rationale, design and methods of a 3-armed randomized controlled trial. Contemp Clin Trials. 2017;57:44-50.

15. Venables E, Ndlovu Z, Munyaradzi D, Martinez-Perez G, Mbofana E, Nyika P, et al. Patient and health-care worker experiences of an HIV viral load intervention using SMS: a qualitative study. PLoS One. 2019;14(4):e0215236.

16. Christopoulos KA, Riley ED, Carrico AW, Tulsky J, Moskowitz JT, Dilworth S, et al. A randomized controlled trial of a text messaging intervention to promote virologic suppression and retention in care in an urban safety-net human immunodeficiency virus clinic: the Connect4Care Trial. Clin Infect Dis. 2018;67(5):751-9.

17. Coleman J, Bohlin KC, Thorson A, Black V, Mechael P, Mangxaba $\mathrm{J}$, et al. Effectiveness of an SMS-based maternal mHealth intervention to improve clinical outcomes of HIV-positive pregnant women. AIDS Care. 2017;29(7):890-7.

18. Pintye J, Rogers Z, Kinuthia J, Mugwanya KK, Abuna F, Lagat H, et al. Two-way short message service (SMS) communication may increase pre-exposure prophylaxis continuation and adherence among pregnant and postpartum women in Kenya. Glob Health Sci Pract. 2020;8(1):55-67.

19. Odeny TA, Bailey RC, Bukusi EA, Simoni JM, Tapia KA, Yuhas $\mathrm{K}$, et al. Text messaging to improve attendance at post-operative clinic visits after adult male circumcision for HIV prevention: a randomized controlled trial. PLoS One. 2012;7(9):e43832.

20. Odeny TA, Bailey RC, Bukusi EA, Simoni JM, Tapia KA, Yuhas $\mathrm{K}$, et al. Effect of text messaging to deter early resumption of sexual activity after male circumcision for HIV prevention: a randomized controlled trial. J Acquir Immune Defic Syndr. 2014;65(2):e50-7.

21. Mayer JE, Fontelo P. Meta-analysis on the effect of text message reminders for HIV-related compliance. AIDS Care. 2017;29(4):409-17.

22. Gross R, Ritz J, Hughes MD, Salata R, Mugyenyi P, Hogg E, et al. Two-way mobile phone intervention compared with standard-ofcare adherence support after second-line antiretroviral therapy failure: a multinational, randomised controlled trial. Lancet Digit Health. 2019;1(1):e26-34.

23. Seidenberg P, Nicholson S, Schaefer M, Semrau K, Bweupe M, Masese N, et al. Early infant diagnosis of HIV infection in Zambia through mobile phone texting of blood test results. Bull World Health Organ. 2012;90(5):348-56.

24. Lester RT, Ritvo P, Mills EJ, Kariri A, Karanja S, Chung MH, et al. Effects of a mobile phone short message service on antiretroviral treatment adherence in Kenya (WelTel Kenya1): a randomised trial. Lancet. 2010;376(9755):1838-45.

25. Shah R, Watson J, Free C. A systematic review and meta-analysis in the effectiveness of mobile phone interventions used to improve adherence to antiretroviral therapy in HIV infection. BMC Public Health. 2019;19(1):915.

26. Joseph-Davey D, Ponce W, Augusto O, Traca D, de Palha de Sousa C. Improved uptake of institutional birth and early infant HIV diagnosis following SMS reminders among PMTCT patients in Mozambique: a randomized control trial. 7th International AIDS Society Conference on HIV Pathogenesis, Treatment and Prevention; June 30-July 3, 2013; Kuala Lampur, Malaysia; 2013.

27. Odeny TA, Hughes JP, Bukusi EA, Akama E, Geng EH, Holmes $\mathrm{KK}$, et al. Text messaging for maternal and infant retention in prevention of mother-to-child HIV transmission services: a pragmatic stepped-wedge cluster-randomized trial in Kenya. PLoS Med. 2019;16(10):e1002924. 
28. Bigna JJ, Noubiap JJ, Kouanfack C, Plottel CS, Koulla-Shiro S. Effect of mobile phone reminders on follow-up medical care of children exposed to or infected with HIV in Cameroon (MORE CARE): a multicentre, single-blind, factorial, randomised controlled trial. Lancet Infect Dis. 2014;14(7):600-8.

29. Smiley SL, Milburn NG, Nyhan K, Taggart T. A systematic review of recent methodological approaches for using ecological momentary assessment to examine outcomes in U.S. based HIV research. Curr HIV/AIDS Rep. 2020;17(4):333-42.

30. Schwebel FJ, Larimer ME. Using text message reminders in health care services: a narrative literature review. Internet Interv. 2018;13:82-104.

31. Finitsis DJ, Pellowski JA, Johnson BT. Text message intervention designs to promote adherence to antiretroviral therapy (ART): a meta-analysis of randomized controlled trials. PLoS One. 2014;9(2):e88166.

32. Coomes CM, Lewis MA, Uhrig JD, Furberg RD, Harris JL, Bann $\mathrm{CM}$. Beyond reminders: a conceptual framework for using short message service to promote prevention and improve healthcare quality and clinical outcomes for people living with HIV. AIDS Care. 2012;24(3):348-57.

33. Kew S. Text messaging: an innovative method of data collection in medical research. BMC Res Notes. 2010;3:342.

34. Johnson D. Collecting data from mHealth users via SMS surveys: a case study in Kenya. Surv Pract. 2016;9:1-8.

35. Amankwaa I, Boateng D, Quansah DY, Akuoko CP, Evans C. Effectiveness of short message services and voice call interventions for antiretroviral therapy adherence and other outcomes: a systematic review and meta-analysis. PLoS One. 2018;13(9):e0204091.

36. The Mobile Money Movement: catalyst to jump-start emerging markets. Innovations: Technology, Governance, Globalization. 2011;6(1):101-17.

37. Gurman TA, Rubin SE, Roess AA. Effectiveness of mHealth behavior change communication interventions in developing countries: a systematic review of the literature. J Health Commun. 2012;17(Suppl 1):82-104.

38. Labrique AB, Kirk GD, Westergaard RP, Merritt MW. Ethical issues in mHealth research involving persons living with HIV/AIDS and substance abuse. AIDS Res Treat. 2013;2013:189645.

39. Connected Women. The Moble gender gap report 2020. GSMA; 2020

40.• Muwonge TR, Ngure K, Katabira E, Mugo N, Kimemia G, Burns $\mathrm{BFO}$, et al. Short message service (SMS) surveys assessing preexposure prophylaxis (PrEP) adherence and sexual behavior are highly acceptable among HIV-uninfected members of serodiscordant couples in East Africa: a mixed methods study. AIDS Behav. 2019;23(5):1267-76. This study provides evidence of the feasibility and acceptability of using SMS surveys to collect data on sexual behavior and adherence to HIV preexposure prophylaxis in sub-Saharan Africa.

41. van der Kop ML, Karanja S, Thabane L, Marra C, Chung MH, Gelmon L, et al. In-depth analysis of patient-clinician cell phone communication during the WelTel Kenyal antiretroviral adherence trial. PLoS One. 2012;7(9):e46033.

42. V Velloza J, Ngure K, Kiptinness C, Quame-Amaglo J, Thuo N, Dew $\mathrm{K}$, et al. A clinic-based tablet application to support safer conception among HIV serodiscordant couples in Kenya: feasibility and acceptability study. Mhealth. 2019;5:4. This study reports on the ability of daily, short SMS surveys to capture sensitive data, including recognition of seasonal variation during end-of-year holidays in sub-Saharan Africa.

43. de Lepper AM, Eijkemans MJ, van Beijma H, Loggers JW, Tuijn CJ, Oskam L. Response patterns to interactive SMS health education quizzes at two sites in Uganda: a cohort study. Tropical Med Int Health. 2013;18(4):516-21.
44. Haberer JE, Kiwanuka J, Nansera D, Wilson IB, Bangsberg DR. Challenges in using mobile phones for collection of antiretroviral therapy adherence data in a resource-limited setting. AIDS Behav. 2010;14(6):1294-301.

45. Devine S, Leeds C, Shlay JC, Leytem A, Beum R, Bull S. Methods to assess youth engagement in a text messaging supplement to an effective teen pregnancy program. J Biomed Inform. 2015;56:379-86.

46. Ballivian A, Azevedo JP, Durbin W. Using mobile phones for high-frequency data collection. In: Toninelli D, Pinter R, de Pedraza P, editors. Mobile research methods: opportunities and challenges of mobile research methodologies. London: Ubiquity Press; 2015. p. 21-39.

47. Kumoji E, Khan SS. Use of short message service for monitoring Zika-related behaviors in four Latin American countries: lessons learned from the field. Mhealth. 2019;5:23.

48. Mehta K, Kumar AMV, Chawla S, Chavda P, Selvaraj K, Shringarpure KS, et al. 'M-TRACK' (mobile phone reminders and electronic tracking tool) cuts the risk of pre-treatment loss to follow-up by $80 \%$ among people living with HIV under programme settings: a mixed-methods study from Gujarat, India. Glob Health Action. 2018;11(1):1438239.

49. Ye M, Kagone M, Sie A, Bagagnan C, Sanou H, Millogo O, et al. Promoting access equity and improving health care for women, children and people living with HIV/AIDS in Burkina Faso through mHealth. J Public Health (Oxf). 2018;40(suppl_2):ii42-51.

50. Hampshire K, Porter G, Mariwah S, Munthali A, Robson E, Owusu SA, et al. Who bears the cost of 'informal mhealth'? Healthworkers' mobile phone practices and associated political-moral economies of care in Ghana and Malawi. Health Policy Plan. 2017;32(1):34-42.

51. Ronen K, Unger JA, Drake AL, Perrier T, Akinyi P, Osborn L, et al. SMS messaging to improve ART adherence: perspectives of pregnant HIV-infected women in Kenya on HIV-related message content. AIDS Care. 2018;30(4):500-5.

52. Curran K, Mugo NR, Kurth A, Ngure K, Heffron R, Donnell D, et al. Daily short message service surveys to measure sexual behavior and pre-exposure prophylaxis use among Kenyan men and women. AIDS Behav. 2013;17(9):2977-85.

53. Drake AL, Begnel E, Pintye J, Kinuthia J, Wagner AD, Rothschild $\mathrm{CW}$, et al. The utility of SMS to report male partner HIV self-testing outcomes among women seeking reproductive health services in Kenya: cohort study. JMIR Mhealth Uhealth. 2020;8(3):e15281.

54. Brown W 3rd, Giguere R, Sheinfil A, Ibitoye M, Balan I, Ho T, et al. Challenges and solutions implementing an SMS text messagebased survey CASI and adherence reminders in an international biomedical HIV PrEP study (MTN 017). J Biomed Inform. 2018;80:78-86.

55. You WX, Comins CA, Jarrett BA, Young K, Guddera V, Phetlhu DR, et al. Facilitators and barriers to incorporating digital technologies into HIV care among cisgender female sex workers living with HIV in South Africa. Mhealth. 2020;6:15.

56. Ossemane EB, Moon TD, Were MC, Heitman E. Ethical issues in the use of SMS messaging in HIV care and treatment in low- and middle-income countries: case examples from Mozambique. J Am Med Inform Assoc. 2018;25(4):423-7.

57. Hirsch-Moverman Y, Daftary A, Yuengling KA, Saito S, Ntoane M, Frederix K, et al. Using mHealth for HIV/TB treatment support in Lesotho: enhancing patient-provider communication in the START study. J Acquir Immune Defic Syndr. 2017;74(Suppl 1):S37-43.

58. Wexler C, Brown M, Hurley EA, Ochieng M, Goggin K, Gautney $\mathrm{B}$, et al. Implementing eHealth technology to address gaps in early infant diagnosis services: qualitative assessment of Kenyan provider experiences. JMIR Mhealth Uhealth. 2018;6(8):e169. 
59. Harrington EK, Drake AL, Matemo D, Ronen K, Osoti AO, JohnStewart G, et al. An mHealth SMS intervention on postpartum contraceptive use among women and couples in Kenya: a randomized controlled trial. Am J Public Health. 2019;109(6):934-41.

60. Unger JA, Ronen K, Perrier T, DeRenzi B, Slyker J, Drake AL, et al. Short message service communication improves exclusive breastfeeding and early postpartum contraception in a low- to middle-income country setting: a randomised trial. BJOG. 2018;125(12):1620-9.

61. Siedner MJ, Santorino D, Haberer JE, Bangsberg DR. Know your audience: predictors of success for a patient-centered texting app to augment linkage to HIV care in rural Uganda. J Med Internet Res. 2015;17(3):e78.

62. Barron P, Peter J, LeFevre AE, Sebidi J, Bekker M, Allen R, et al. Mobile health messaging service and helpdesk for South African mothers (MomConnect): history, successes and challenges. BMJ Glob Health. 2018;3(Suppl 2):e000559.

63. Lester RT, Mills EJ, Kariri A, Ritvo P, Chung M, Jack W, et al. The HAART cell phone adherence trial (WelTel Kenya1): a randomized controlled trial protocol. Trials. 2009;10:87.

64. Mitra P. Challenges and opportunities for mHealth in Kenya 2014 [Available from: www.techchange.org/2014/11/03/mhealthchallenges-opportunities-kenya-india/. Accessed 27 June 2020.

65. Njoroge M, Zurovac D, Ogara EA, Chuma J, Kirigia D. Assessing the feasibility of eHealth and mHealth: a systematic review and analysis of initiatives implemented in Kenya. BMC Res Notes. 2017;10(1):90.

66. Begnel ER, Escudero J, Mugambi M, Mugwanya K, Kinuthia J, Beima-Sofie K, et al. High pre-exposure prophylaxis awareness and willingness to pay for pre-exposure prophylaxis among young adults in Western Kenya: results from a population-based survey. Int J STD AIDS. 2020;31(5):454-9.

67. Sudhinaraset M, Landrian A, Afulani PA, Diamond-Smith N, Golub G. Association between person-centered maternity care and newborn complications in Kenya. Int J Gynaecol Obstet. 2020;148(1):27-34.

68. Al-Shammari I, Roa L, Yorlets RR, Akerman C, Dekker A, Kelley $\mathrm{T}$, et al. Implementation of an international standardized set of outcome indicators in pregnancy and childbirth in Kenya: utilizing mobile technology to collect patient-reported outcomes. PLoS One. 2019;14(10):e0222978.

69. Bardosh KL, Murray M, Khaemba AM, Smillie K, Lester R. Operationalizing mHealth to improve patient care: a qualitative implementation science evaluation of the WelTel texting intervention in Canada and Kenya. Glob Health. 2017;13(1):87.

70. Wahl B, Cossy-Gantner A, Germann S, Schwalbe NR. Artificial intelligence (AI) and global health: how can AI contribute to health in resource-poor settings? BMJ Glob Health. 2018;3(4):e000798.
71. Catania JA, Gibson DR, Chitwood DD, Coates TJ. Methodological problems in AIDS behavioral research: influences on measurement error and participation bias in studies of sexual behavior. Psychol Bull. 1990;108(3):339-62.

72. Kelly CA, Soler-Hampejsek E, Mensch BS, Hewett PC. Social desirability bias in sexual behavior reporting: evidence from an interview mode experiment in rural Malawi. Int Perspect Sex Reprod Health. 2013;39(1):14-21.

73. Langhaug LF, Sherr L, Cowan FM. How to improve the validity of sexual behaviour reporting: systematic review of questionnaire delivery modes in developing countries. Tropical Med Int Health. 2010;15(3):362-81.

74. Greenleaf AR, Gibson DG, Khattar C, Labrique AB, Pariyo GW. Building the evidence base for remote data collection in low- and middle-income countries: comparing reliability and accuracy across survey modalities. 2017;19(5):e140.

75. Phillips AE, Gomez GB, Boily M-C, Garnett GP. A systematic review and meta-analysis of quantitative interviewing tools to investigate self-reported HIV and STI associated behaviours in low- and middle-income countries. Int J Epidemiol. 2010;39(6):1541-55.

76. Cleland J, Boerma JT, Carael M, Weir SS. Monitoring sexual behaviour in general populations: a synthesis of lessons of the past decade. Sex Transm Infect. 2004;80(suppl 2):ii1.

77. Drake A, Kinuthia J, Wagner A, Rothschild C, Abuna F, Kemunto V, et al. Short message service (SMS) enhances capture of male partner HIV self-testing outcomes among women seeking reproductive health services in Kenya. JMIR mHealth and uHealth. 2019;8(3):e15281

78. Simoni JM, Ronen K, Aunon FM. Health behavior theory to enhance eHealth intervention research in HIV: rationale and review. Curr HIV/AIDS Rep. 2018;15(6):423-30.

79. Hall AK, Cole-Lewis H, Bernhardt JM. Mobile text messaging for health: a systematic review of reviews. Annu Rev Public Health. 2015;36:393-415.

80. Head KJ, Noar SM, Iannarino NT, Grant Harrington N. Efficacy of text messaging-based interventions for health promotion: a metaanalysis. Soc Sci Med. 2013;97:41-8.

81. Park LG, Howie-Esquivel J, Dracup K. A quantitative systematic review of the efficacy of mobile phone interventions to improve medication adherence. J Adv Nurs. 2014;70(9):1932-53.

Publisher's Note Springer Nature remains neutral with regard to jurisdictional claims in published maps and institutional affiliations. 